\title{
Counselling women with congenital cardiac disease
}

They should be empowered to make informed choices

\author{
Matthew Cauldwell clinical research fellow ${ }^{1}$, Philip J Steer emeritus professor of obstetrics ${ }^{1}$, Mark \\ Johnson professor of obstetrics ${ }^{1}$, Michael Gatzoulis professor of cardiology ${ }^{2}$
}

\author{
${ }^{1}$ Academic Department of Obstetrics and Gynaecology, Imperial College London, London SW10 9NH, UK; ${ }^{2}$ National Heart and Lung Institute, \\ Imperial College London
}

Preconception counselling is particularly important for women with congenital heart disease because these conditions are the leading direct cause of maternal death in many developed countries, including the United Kingdom. ${ }^{1}$ Recent developments in medicine and the law require us to re-evaluate how preconception counselling is delivered.

The principle of patient autonomy has superseded the traditional paternalistic model of healthcare, with shared decision making the new standard. This involves agreeing the evidence base during the clinical consultation (placing an imperative on the doctor to get the facts right) and joint decision making, taking account of the patient's preferences and values. Decision support tools facilitate sharing of complex information in some disciplines; however, no such tools exist for heart disease and pregnancy, so clinicians have to rely on their personal knowledge and counselling skills.

Communicating risk is a challenge in preconception counselling in congenital heart disease. Pregnancy increases the risk of mortality and morbidity in women, ${ }^{2}$ but the data on the extent of these risks are flawed. Studies are generally observational, retrospective, and small. For example, patients with Marfan syndrome with an aortic root of $<40 \mathrm{~mm}$ are often quoted a risk of dissection in pregnancy of about $1 \%$; but all relevant studies include fewer than 100 patients, managed with various treatment regimens. ${ }^{3}$ Larger, better designed, and ideally prospective studies are needed. Another challenge is that understanding of risk is subjective and interpretation varies among individuals. ${ }^{4}$ The UK Supreme Court Montgomery ruling states the "onus should not be upon the patient, who may not know that there is anything to ask about." ${ }^{5}$ Clinicians must discuss risks fully in a sensitive manner, aiming to inform but not frighten unnecessarily, and state that there is always some uncertainty with regard to risk.

\section{Is preconception counselling effective?}

Preconception counselling is criticised because there is a shortage of evidence showing that it improves patient outcomes. ${ }^{6}$ Furthermore, we do not routinely check whether women feel empowered by counselling. Making value judgments about its success is difficult. From the outset clinicians must not only try to put themselves in the patient's shoes and ask themselves what they think the patient ought to know but also establish the patient's priorities and what she wishes to know. We need to find out whether what we tell women helps them to come to decisions that are right for them. Effectiveness cannot be measured by simply trying to eliminate poor pregnancy outcomes associated with congenital heart disease. Prescribing pregnancy aversion therapy would reduce such outcomes but is not practical or ethical.

For women with more complex conditions such as a Fontan circulation preconception counselling has an additional dimension. Their clinical condition will inevitably deteriorate over time, ${ }^{7}$ and women need to know that the risks of pregnancy will be lower at a younger age, when they are still relatively free from the adverse effects of their condition, than when they are older. Only they can decide how to respond to these risks. Our data show the average age at pregnancy in women with cardiac disease is similar to that in the general population, suggesting social factors may be more important than medical concerns in timing of pregnancy (unpublished data).

Preconception counselling should not be aimed at discouraging women from becoming pregnant; rather it should empower them to make informed choices. We need to understand better the effects of heart disease on pregnancy and so be able to provide more accurate information on the long term effects of pregnancy on heart disease. Although current evidence suggests that pregnancy does not impair cardiovascular prospects for the medium to long term in many cardiac conditions, ${ }^{8}$ adverse cardiovascular remodelling may occur. ${ }^{9}$ Above all, we need to ensure timely access to counselling and involve patients in designing a process that best meets their needs.

Competing interests: We have read and understood BMJ policy on declaration of interests and have no relevant interests to declare. Provenance and peer review: Not commissioned; externally peer reviewed. 
1 MBRRACE-UK. Savings lives, improving, mothers' care. 2014. www.npeu.ox.ac.uk downloads/files/mbrrace-uk/reports/Saving\%20Lives\%20Improving\%20Mothers\%20Care\% 20report\%202014\%20Full.pdf. 2015.

2 Roos-Hesselink JW, Ruys TP, Stein JI, et al. ROPAC Investigators. Outcome of pregnancy in patients with structural or ischaemic heart disease: results of a registry of the European Society of Cardiology. Eur Heart J 2013;34:657-65. doi:10.1093/eurheartj/ ehs270. 22968232

3 Lind J, Wallenburg HC. The Marfan syndrome and pregnancy: a retrospective study in a Dutch population. Eur J Obstet Gynecol Reprod Biol 2001;98:28-35. doi:10.1016/S03012115(01)00314-1. 11516796

Adelsward V, Sachs L. Risk discourse: recontextualization of numerical values in clinical practice. Text 1998;18:191-210doi:10.1515/text.1.1998.18.2.191.

5 Montgomery (Appellant) v Lanarkshire Health Board (Respondent) (Scotland) [2015] UKSC 11) https://www.supremecourt.uk/decided-cases/docs/UKSC_2013 0136 Judgment. pdf
6 Shannon GD, Alberg C, Nacul L, Pashayan N. Preconception healthcare and congenital disorders: systematic review of the effectiveness of preconception care programs in the prevention of congenital disorders. Matern Child Health J 2014;18:1354-79. doi:10.1007/ prevention of congenital disorders.

7 Pundi KN, Johnson JN, Dearani JA, et al. 40-year follow-up after the Fontan operation: long-term outcomes of 1,052 patients. J Am Coll Cardiol 2015;66:1700-10. doi:10.1016/ j.jacc.2015.07.065. 26449141.

8 Uebing A, Arvanitis $\mathrm{P}, \mathrm{Li} \mathrm{W}$, et al. Effect of pregnancy on clinical status and ventricular function in women with heart disease. Int J Cardiol 2010;139:50-9. doi:10.1016/j.jijcard. function in women with

9 Guédès A, Mercier LA, Leduc L, Bérubé L, Marcotte F, Dore A. Impact of pregnancy on the systemic right ventricle after a Mustard operation for transposition of the great arteries. J Am Coll Cardiol 2004;44:433-7. doi:10.1016/j.jacc.2004.04.037. 15261944.

Published by the BMJ Publishing Group Limited. For permission to use (where not already granted under a licence) please go to http://group.bmj.com/group/rights-licensing/ permissions 